\title{
Study on the Authenticity of Identification Methods in White Spirit by Infrared Spectrometer
}

\author{
Dekui Bai ${ }^{1,2}$, Ni Wang ${ }^{2}$, Quanhong Ying ${ }^{2}$, Jinhui Lin ${ }^{1}$ \\ ${ }^{1}$ College of Material \& Chemical Engineering Chengdu University of Technology, Chengdu, China \\ ${ }^{2}$ Mianyang Product Quality Supervision and Inspection Bureau, Mianyang, China \\ Email: bdk123123@126.com, wangni0308@126.com
}

Received July 2013

\begin{abstract}
This experiment studies on the used infrared spectroscopy to establish technology methods for liquor identification methods, as well as offers the science data for establishment of the fingerprint in white spirit. The results have shown that using near-infrared spectroscopy analysis of liquor has the obvious features such as strong specificity, good reproducibility, simple operation, and finally confirmed that it is an authentic and ideal method for identification in white spirit.
\end{abstract}

Keywords: Infrared Spectrometer (IR); White Spirit; Authenticity Identification

\section{Introduction}

Near-infrared light was between visible light and infrared light and electromagnetic waves, the American Society for Testing Materials (ASTM) defines that nearly infrared spectral region is a wavelength range from $780 \mathrm{~nm}$ to $526 \mathrm{~nm}$. Almost customary divisions are divided into short-wavelength near-infrareds (780 - $1100 \mathrm{~nm})$ and long wave near infrareds (1100 - $2526 \mathrm{~nm})[1,2]$.

Since in the 1950s of the 20th century, near infrared spectroscopy was applied in analysis of agricultural and sideline products, but it was limited by technology and slow pace of development in the next 30 years. While after the 1980s of the 20th century, with the rapid development of computer technology and chemical methods in the context of spectral information extraction and elimination, the background interference achieved good results, as well as near-infrared spectroscopy in testing technical characteristics that are unique and the understanding of near-infrared spectroscopy for its wide range of applications [2-7].

Through the study of near-infrared technology, we understand that currently most applications of near-infrared technology in Chinese herbal medicines, as well as indications of food analysis and studies have reported the use of near-infrared spectrum detection models, as well as Chinese medicinal materials for the identification of Chinese medicinal materials [8-10]. Therefore, we will use IR to analyze the liquor, to further develop the liquor fingerprint spectrum database in order to identify method and provide technical support to establish authenticity of liquor.

\section{Experimental Method}

\subsection{Instrument}

FOSS Company's NIR Analyzer (NIRS DS 2500), Instrument acquisition parameters listed in Table 1.

\subsection{Experiment Subject}

In accordance with the production lot, year of production, the production month difference, choice the maotai-lavor liquor as a test sample. Experiment on the production of 2009-2012 years month 16 sets of samples, plus the control sample 1 group (No.17), details are shown in Table 2.

\subsection{Experimental Process}

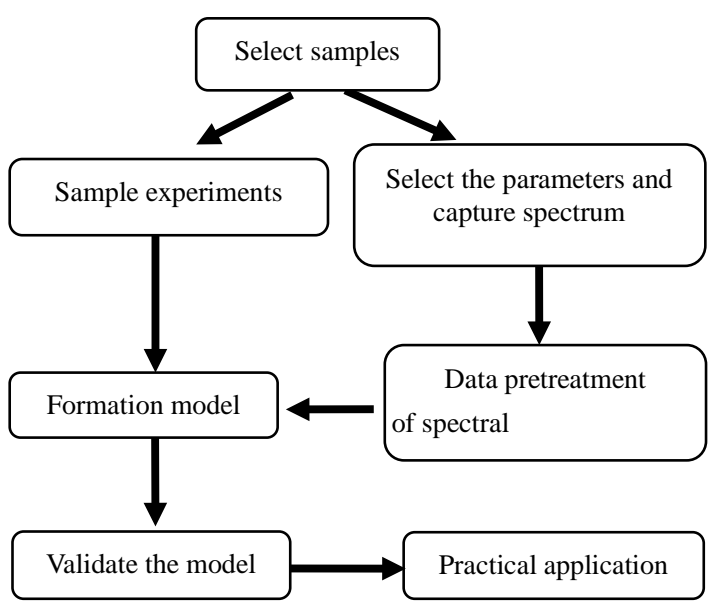


Table 1. Instrument acquisition parameters.

\begin{tabular}{cc}
\hline Item & Parameter \\
\hline Signal Energy & $5 \mathrm{~V}$ \\
Resolution & $2 \mathrm{~cm}^{-1}$ \\
Scanning Frequency & 32 \\
Spectral Range & $400-2500 \mathrm{~cm}^{-1}$ \\
Reference & Air \\
Spectrum Format & Absorbance \\
\hline
\end{tabular}

Table 2. Sample information sheet.

\begin{tabular}{cccc}
\hline NO. & Manufacturing Codes & NO. & Manufacturing Codes \\
\hline 1 & 20090803 & 10 & 20110823 \\
2 & 20091022 & 11 & 20111015 \\
3 & 20091223 & 12 & 20111217 \\
4 & 20100405 & 13 & 20120108 \\
5 & 20100611 & 14 & 20120327 \\
6 & 20101106 & 15 & 20120704 \\
7 & 20101214 & 16 & 20121024 \\
8 & 20110105 & 17 & 20121218 \\
9 & 20110129 & & \\
\hline
\end{tabular}

\section{Results and Discussion}

\subsection{Spectrum Pretreatment}

Pretreatment to the original spectrum's aim is to use mathematical methods to minimize the impact of noise signals, thereby increasing the accuracy and reliability of the model, spectral pretreatment mainly to solve the noise filtering, data filtering, optimization of the spectral range and eliminate the effects of other factors to the light spectrum information, correction of laying the foundations for modeling and prediction of unknown samples [11-13].

\section{Noise Remove}

Noise mainly comes from the high frequency random noise and baseline drift, the sample is not uniform, light scattering, etc. Because of the influence of the noise, the useful information of the component under test is hard to stand out, and the model precision, the near infrared spectral analysis of the original spectrum can use the following methods to remove.

1) Smooth processing. Smoothing is a common way to filter out noise, and its main function is to improve the signal-to-noise ratio, the spectrum of removing high frequency noise interference of the signal. Currently used
Savitzky-Golay smoothing function on near-infrared spectroscopy, as a result of smoothing, high frequency noise is reduced, spectrum of random scattered, but it cannot be completely eliminated by Fourier transform filtering, analysis of optical Fourier transform spectrum signal before dropping the transformed frequency, inverse transform back to the status quo ante, and then participate in the return calculation.

2) Wavelet transforms. Spectrum denoising method based on wavelet transform: wavelet transforms the original spectrum, by wavelet coefficients $\omega$, according to Min value method will certainly factor $\omega$ small coefficients in weakened or removed, be $\omega_{\text {den }}$ " under the reconstruction when the noise filtering of signals.

3) Baseline correction treatment of the most common method is to first order differential and second order differential. Differential spectroscopy can enhance the original signal spectrum, so that will help in complex in peak shape to better identify exactly where the peak, so as to achieve the purpose of the differential spectra. First order differential can eliminate baseline shift, secondorder differential can eliminate baseline drift, and this is because the second derivative of the line is zero.

First derivative: $y_{i}^{\prime}=y_{i+g}-y_{i-g}$

Second derivative: $y_{i}^{\prime}=y_{i+2 g}-2 y_{i}+y_{i-2 g}$

$g$ was spectral interval, size can be set according to the circumstances.

\subsection{Standard-Normalized}

Near-infrared spectroscopy, sample homogeneity, particle size and length of the light path also often affect the shape of the spectrum, the spectrum standard normalization process is the most ideal solution measuring changes in the optical path used to remove light change or specimen dilution effects, such as changes to the light spectrum produced. Spectrum under a of method has three species: minimum/maximum under a of, and vector under a of, and back zero correction, which common of is vector under a of it is first calculation out spectrum of sucking photometric average, again runs out spectrum minus the average, such spectrum of in the value for zero, calculate all of sucking photometric of sq and, then runs out spectrum divided by the sq and of sq root, results spectrum of vector under a of is 1 . Back to the zero calibration is run out of the absorbance spectrum minus the minimum makes smallest absorbance reaches zero.

\subsection{Multiple Scatter Correction}

Transmission spectral measurements of multiple scattering correction not only fixes errors in the optical path, but also can eliminate reflection spectroscopy and diffusion in the transmission spectra of light scattering effect, multiple scattering correction algorithm is as follows: 
a) Calculate the average of all sample spectra:

$$
\bar{A}_{j}=\frac{1}{n} \sum_{i=1}^{n} A_{i, j}
$$

b) Calculation of each sample spectra with an average spectrum to the linear regression, regression coefficient is obtained mi, $b_{i}$ :

$$
A_{i}=m_{i} \bar{A}+b_{i}
$$

c) Calculate calibration spectra:

$$
A_{i(M S C)}=\frac{\left(A_{i}-b_{i}\right)}{m_{i}}
$$

Formula, $i=1,2 \cdots \mathrm{n}$. As the number of samples; $j$ represents the number of $j$-wave.

\subsection{Modeling}

Basic principles and methods of near-infrared spectroscopy technology development at the same time, chemical metrology research in the 1970s of the 20th century, combining knowledge of mathematics, statistics and computer science. Study on extract information from data, one of the most important developments is the use of factor analysis, that is, with a large amount of data for coordinate transformation in order to achieve the objective of reduced dimensions, which is to eliminate the overlapping information coexistence. Typical applications such as principal component analysis: spectral data by principal component analysis, again and find the correction factor dependent variable regression, principal component regression based on partial least-squares regression of development, the matrix for dimension reduction methods in light spectrum while introducing variable information.

Thus, near-infrared spectroscopy, including near-in- frared spectrometry and chemical software and application model of three-part, three combination to meet the technical requirements for the rapid analysis, mathematical modeling methods are the main research focuses.

\subsection{Data-Processing Software}

1) TQ Analystv6 software. The software is United States heat with high power company a definitive spectral analysis software, offers a variety of quantitative and qualitative analyses for spectral analyses and modeling methods. The software provides quantitative modeling of progressive multiple linear regression, principal component regression and partial least squares regression. Qualitative modeling method matches the distance discriminate analysis, discriminate partial least squares. When modeling, first select the algorithms, software will appear in the window with the algorithm parameters and associated information, in accordance with the requirements set, and to establish the appropriate quantitative or qualitative models.

2) MATLAB. MATLAB is the Math works company that has developed a set of calculations, graph visualization and editing functions in one powerful, easy math application software. The software supports the quantitative and qualitative modeling analysis and 3D graphics drawing, etc.

\subsection{Orientation Analysis Results}

To test samples for infrared spectrometry, results are detailed in Figures 1 - 17.

Experimental sample (Figures 1 - 16) test results compared to the control sample (Figure 17(e)) results concluded that: although with a response at the same wavelength of Infrared Spectra, control sample peak height is significantly below the peak of authenticity. In

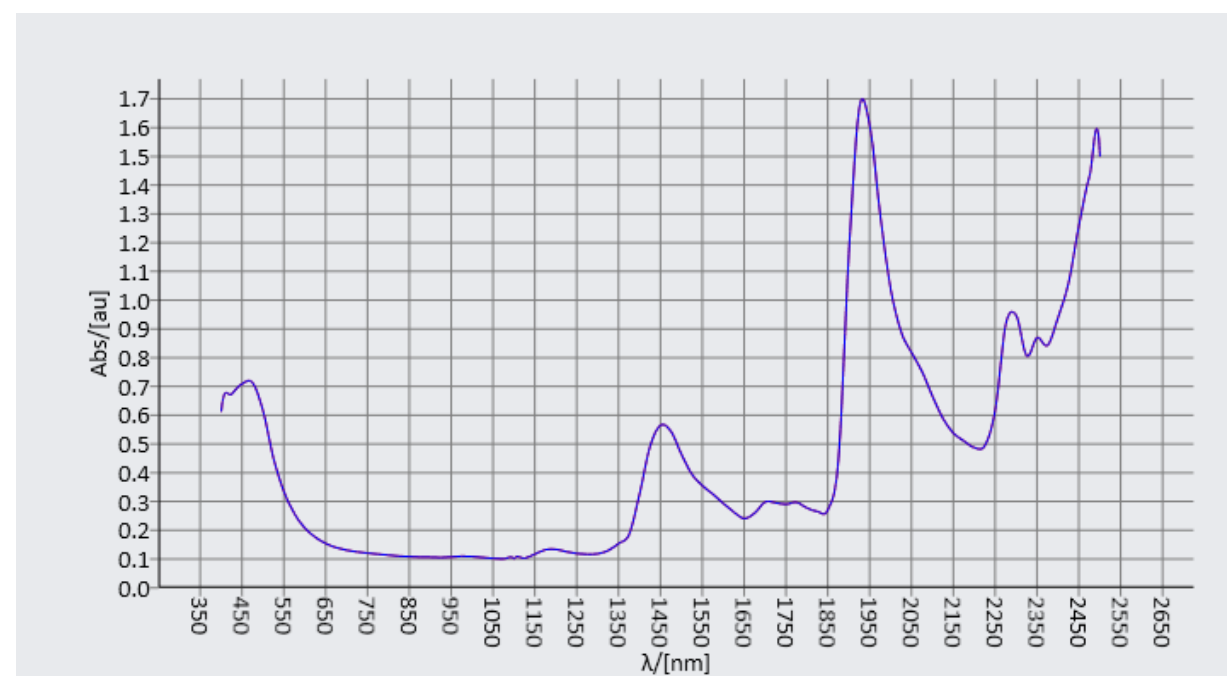

Figure 1. No.20100611 sample spectra. 


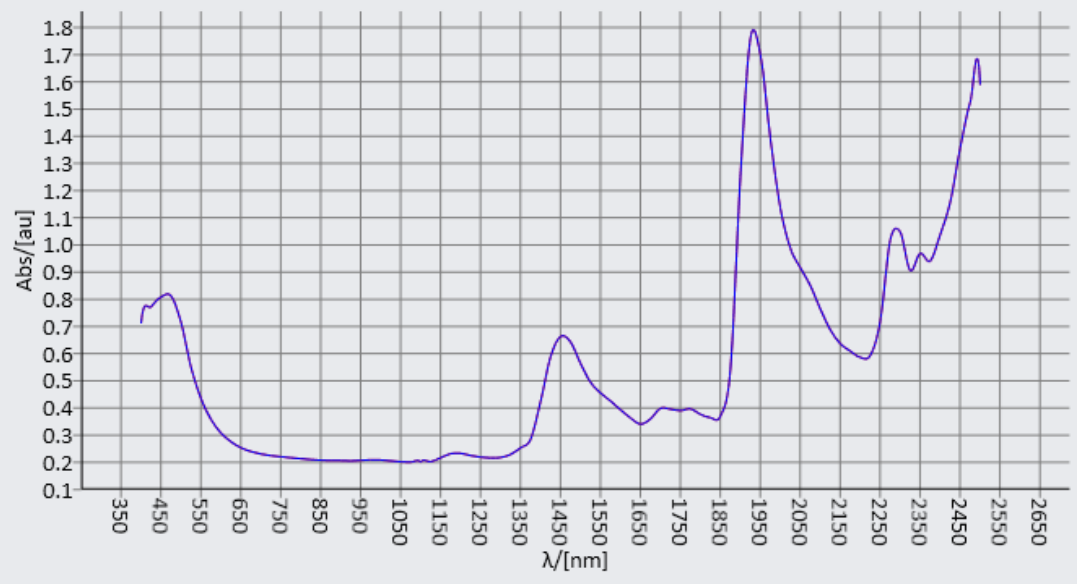

Figure 2. No. 20091022 sample spectra.

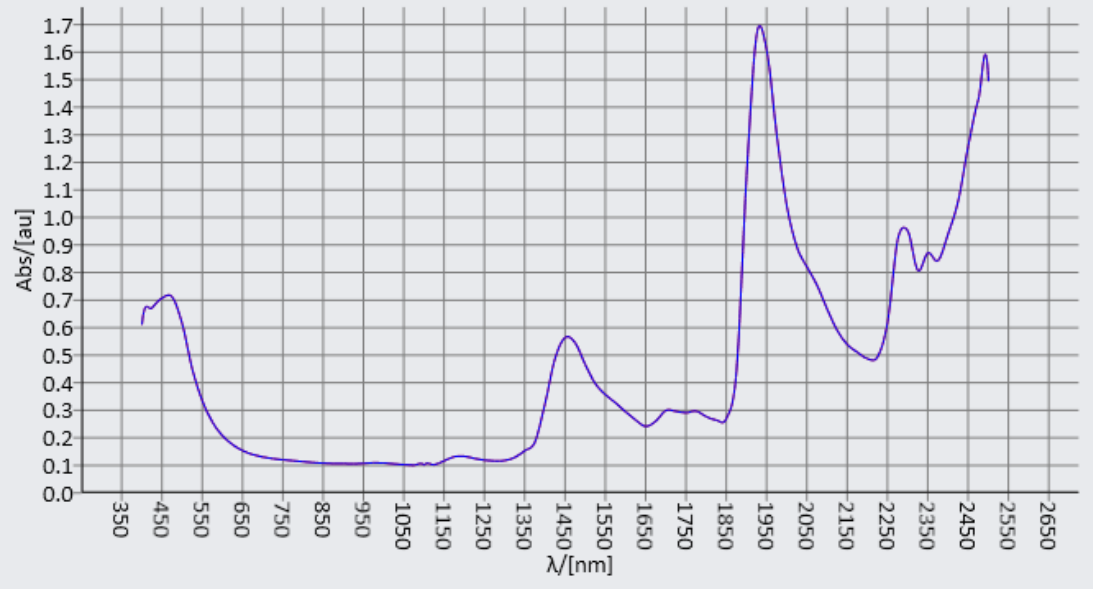

Figure 3. No. 20101214 sample spectra.

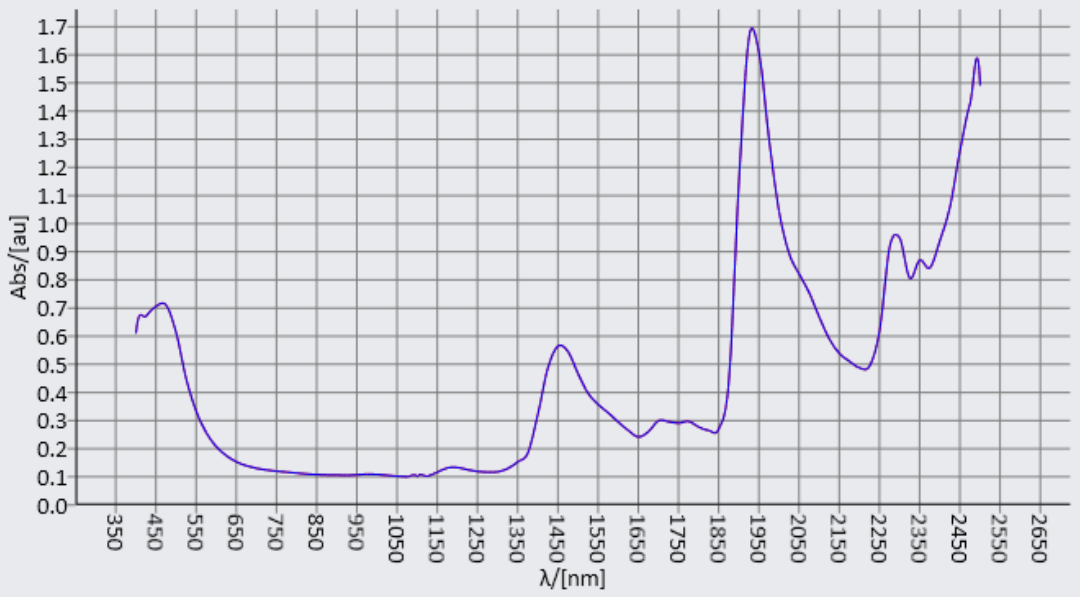

Figure 4. No. 20091223 sample spectra. 


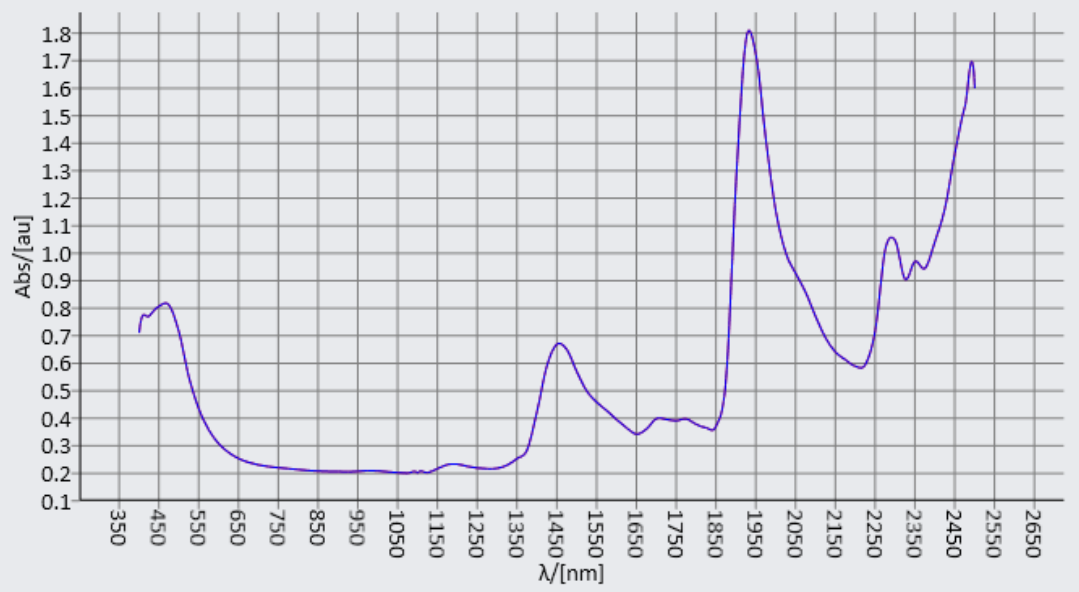

Figure 5. No. 20110105 sample spectra.

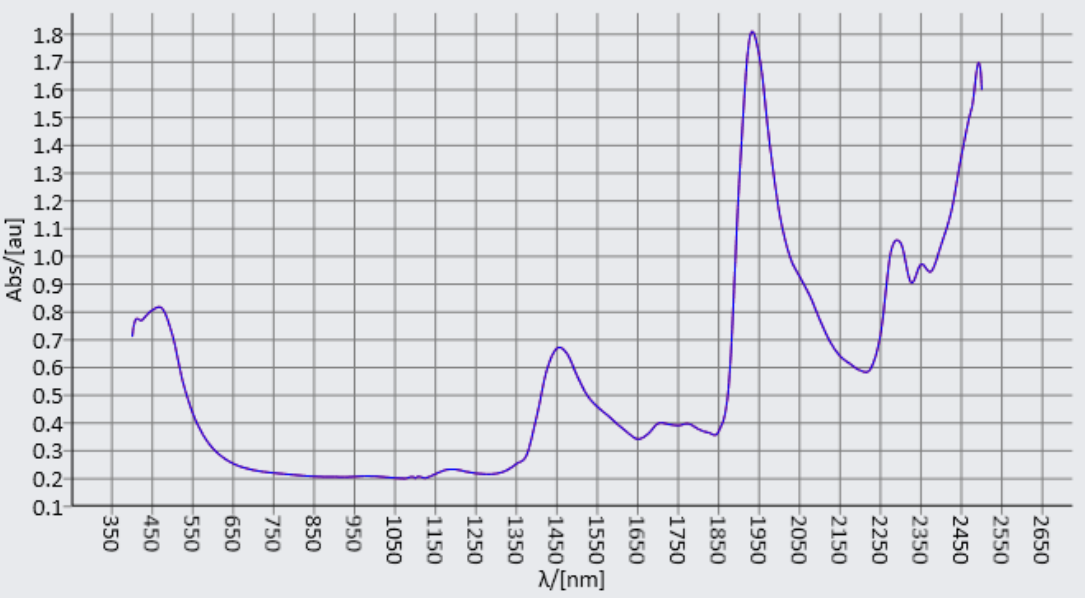

Figure 6. No. 20100405 sample spectra.

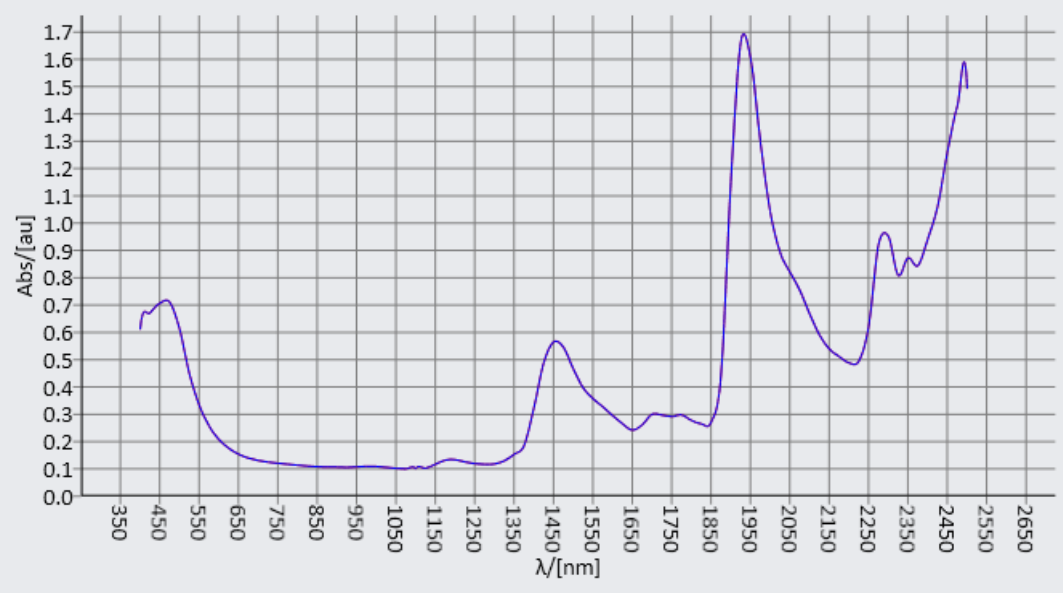

Figure 7. No. 20110823 sample spectrum. 


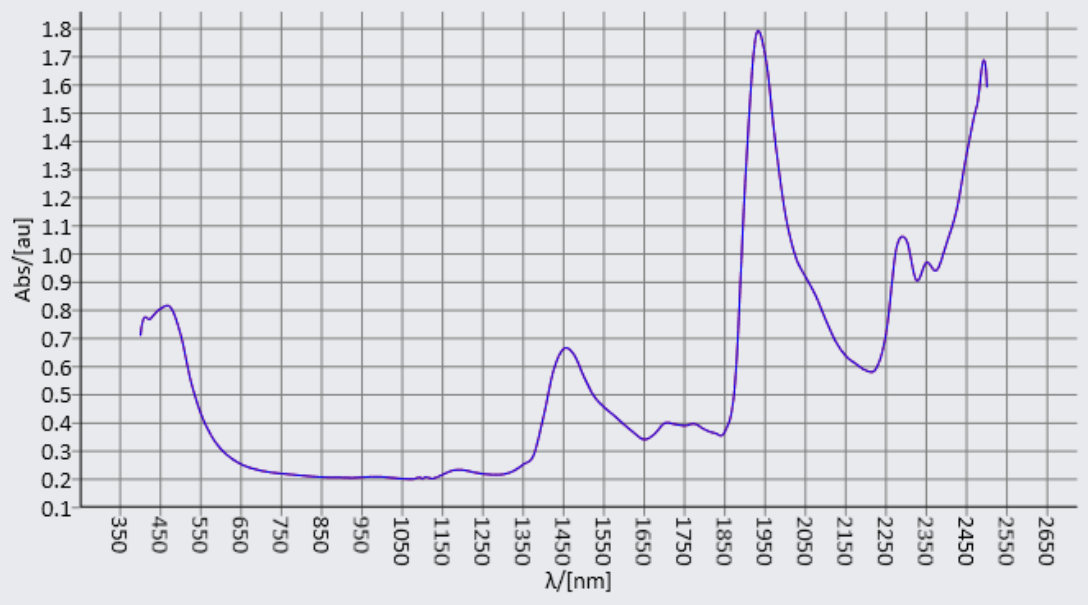

Figure 8. No. 20101106 sample spectra.

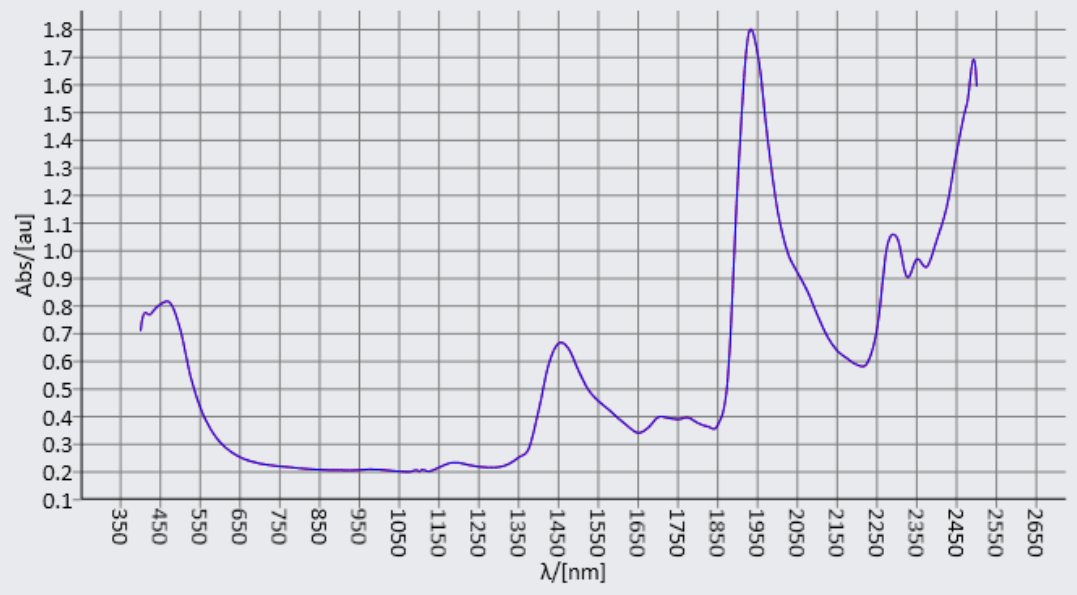

Figure 9. No. 20111015 sample spectra.

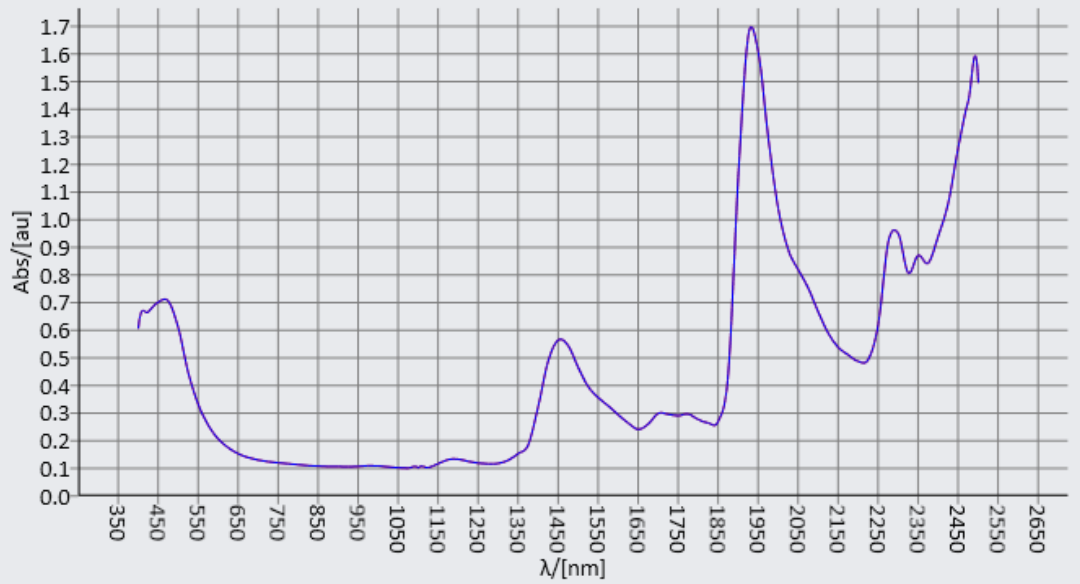

Figure 10. No. 20111217 sample spectra. 


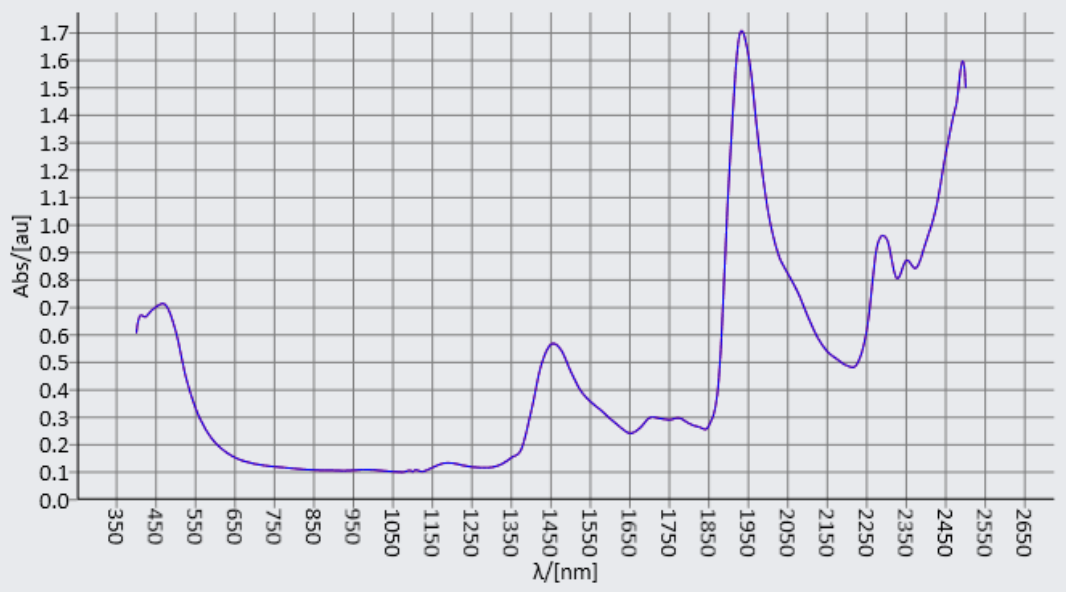

Figure 11. No. 20120327 sample spectra.

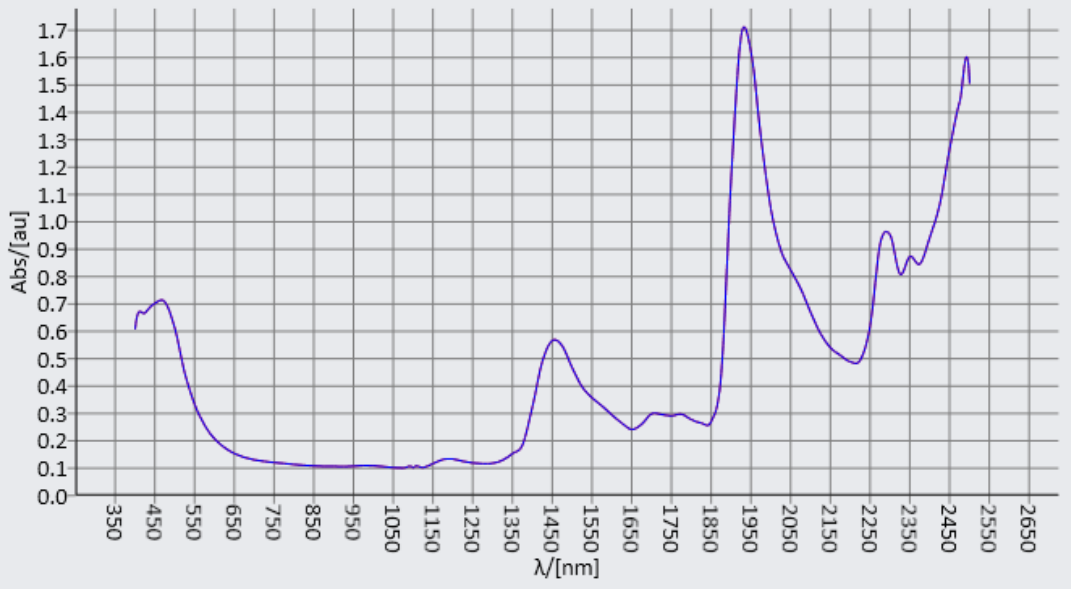

Figure 12. No. 20120108 sample spectra.

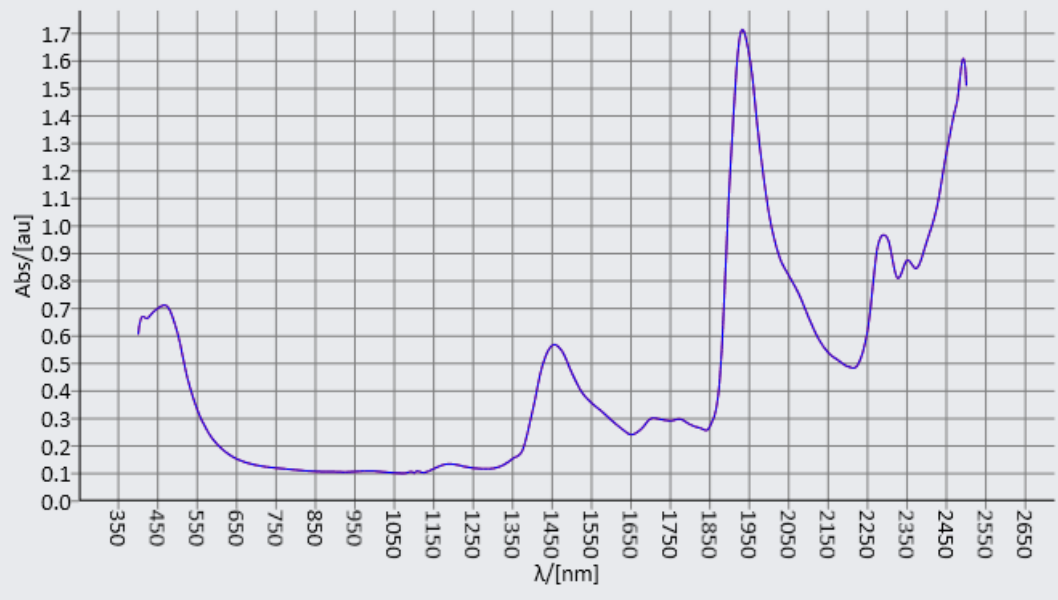

Figure 13. No. 20090803 sample spectrum. 


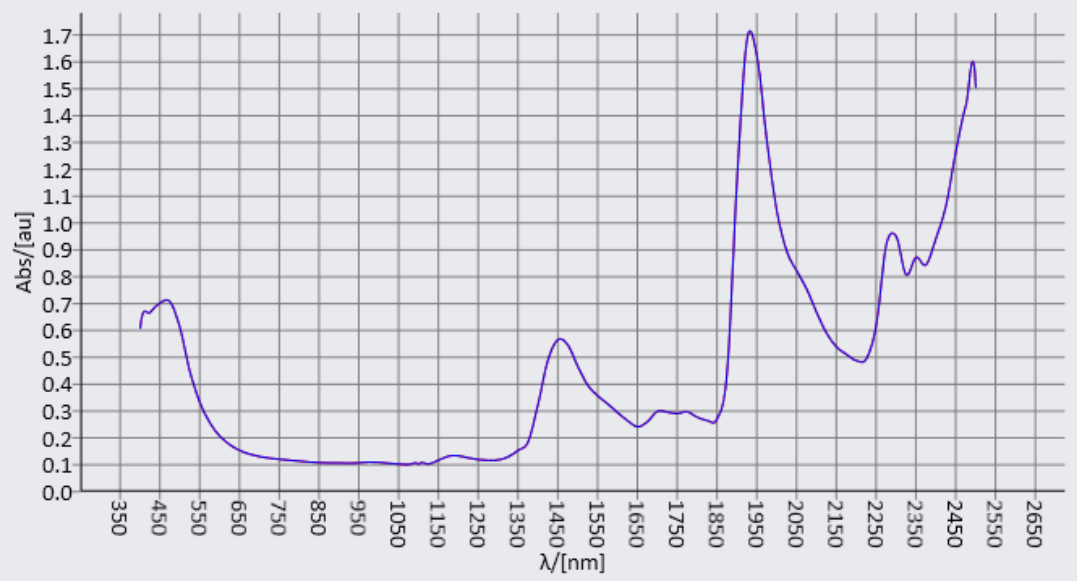

Figure 14. No. 20120704 sample spectra.

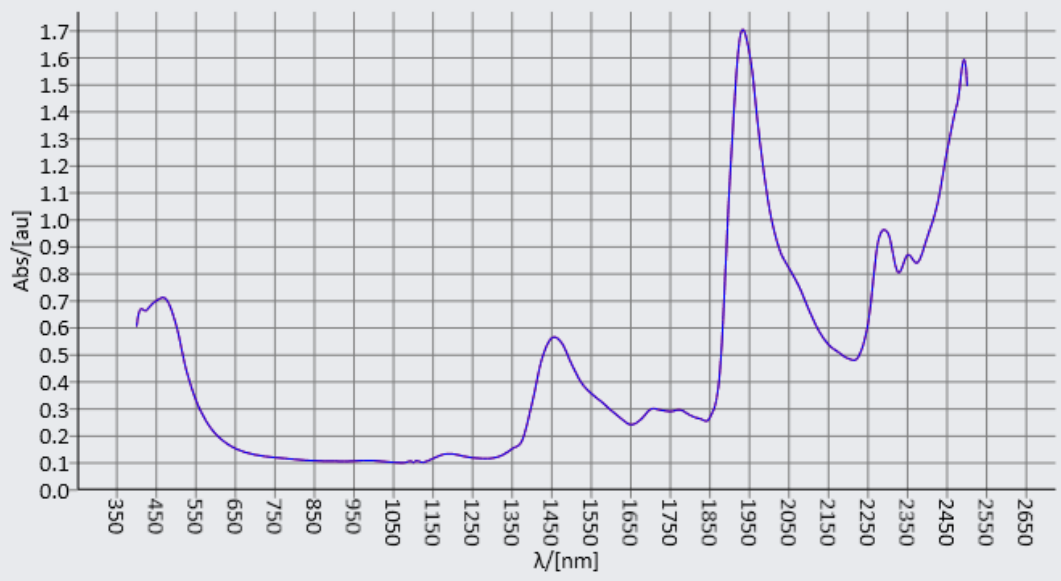

Figure 15. No. 20110129 sample spectra.

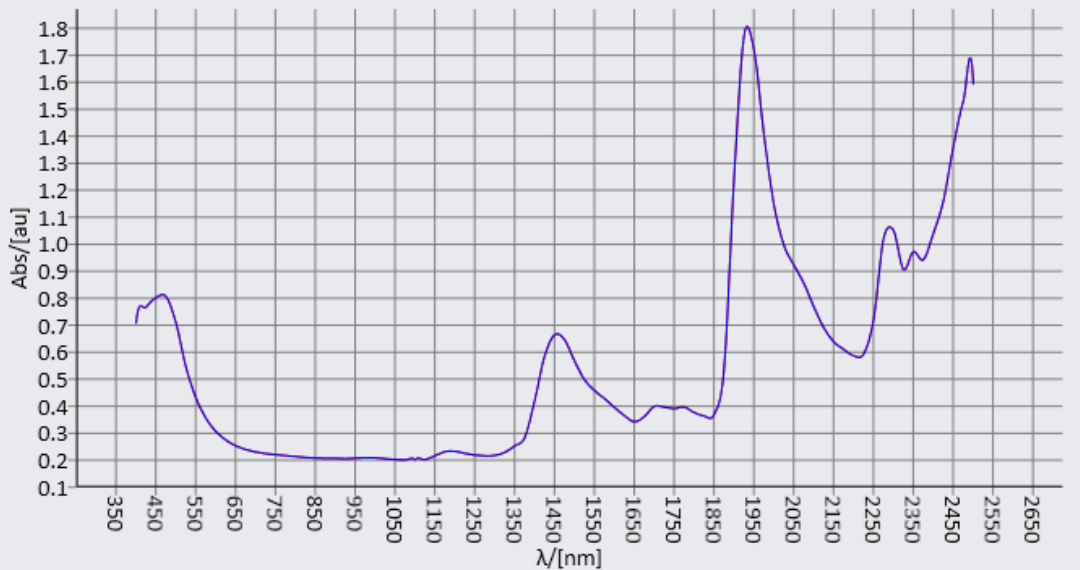

Figure 16. No. 20121024 sample spectra. 


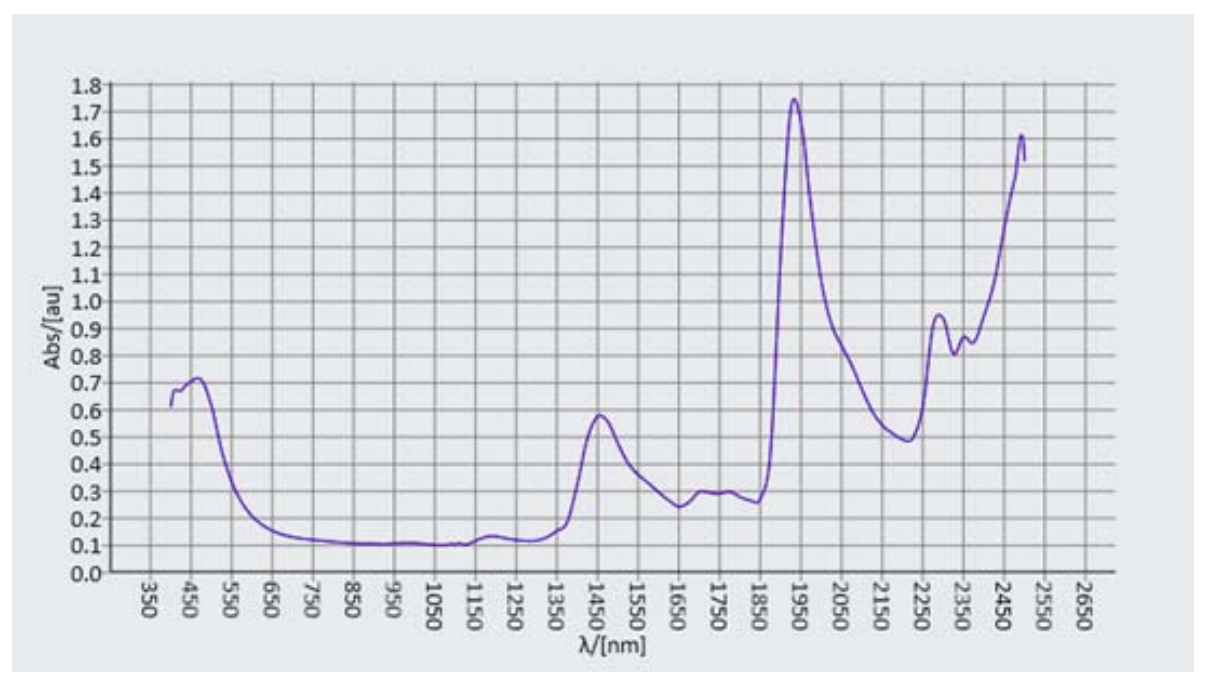

Figure 17. The comparison sample spectra.

addition, according to the experimental sample analyzed by IR spectra, IR spectra of all samples approximate overlapping, has certain regularity, so completely to the modeling and identification of authenticity of liquor degrees.

\subsection{Quantitative Analysis}

With reference to the current liquor class national standard method, selected ethyl caproate, alcohol, methanol, ethyl acetate four parameters for quantitative analysis, detailed in Table 3 and Figures 18 to 21.

Figures 18-21, with sample number in Table 3 as abscissa, experimental results to ordinate to draw. Sample number 1 - 16 for the experimental samples of authentic, 17th for the control sample, and average as shown in the figure to the arithmetic mean of the sample of authenticity. Quantitative experimental results indicate that all experimental contents of the parameters in the sample the real thing is a certain range of fluctuations, overall changed little, however the control sample the obvious deviation from the true value (Average value).

\section{Conclusions}

In this article, infrared spectroscopy applied to liquor of identifying the authenticity of degrees have succeeded in establishing analysis model, and carrying out a qualitative and quantitative analysis of the simple. Research results show that used infrared spectroscopy to establish authenticity liquor identification data models is practical, real simple, and fast. Successful application of this technology for the supervision of government departments, manufacturers provide strong technical support.

We should bear in mind that the technical key points in the process are: 1) The stability of the sample. Liquor volatile $\rightarrow$ volatilize at room temperature, so on the choice of experimental study to comprehensive consideration of
Table 3. Quantitative analysis results.

\begin{tabular}{ccccc}
\hline $\begin{array}{c}\text { Manufacturing } \\
\text { Codes }\end{array}$ & $\begin{array}{c}\text { Ethyl caproate } \\
(\mathrm{g} / \mathrm{L})\end{array}$ & $\begin{array}{c}\text { Alcohol } \\
(\% \mathrm{vol})\end{array}$ & $\begin{array}{c}\text { Methanol } \\
(\mathrm{g} / \mathrm{L})\end{array}$ & $\begin{array}{c}\text { Ethyl Acetate } \\
(\mathrm{g} / \mathrm{L})\end{array}$ \\
\hline 20100611 & 2.12 & 53.77 & 0.15 & 0.87 \\
20100405 & 2.15 & 54.12 & 0.15 & 0.87 \\
20101214 & 2.18 & 54.12 & 0.15 & 0.87 \\
20101106 & 2.21 & 54.00 & 0.16 & 0.87 \\
20110105 & 2.18 & 53.26 & 0.13 & 0.86 \\
20120108 & 2.17 & 53.31 & 0.13 & 0.86 \\
20110823 & 2.28 & 54.12 & 0.16 & 0.87 \\
20120704 & 2.26 & 54.11 & 0.16 & 0.87 \\
20111015 & 2.20 & 53.78 & 0.16 & 0.86 \\
20121024 & 2.24 & 54.14 & 0.16 & 0.87 \\
20120327 & 2.20 & 53.92 & 0.16 & 0.87 \\
20091022 & 2.23 & 53.96 & 0.16 & 0.86 \\
20090803 & 2.25 & 54.23 & 0.18 & 0.87 \\
20091223 & 2.28 & 54.03 & 0.18 & 0.87 \\
20110129 & 2.19 & 54.35 & 0.14 & 0.87 \\
20111217 & 2.23 & 54.27 & 0.14 & 0.87 \\
Compare Sample & 2.10 & 51.56 & 0.18 & 0.90 \\
\hline
\end{tabular}

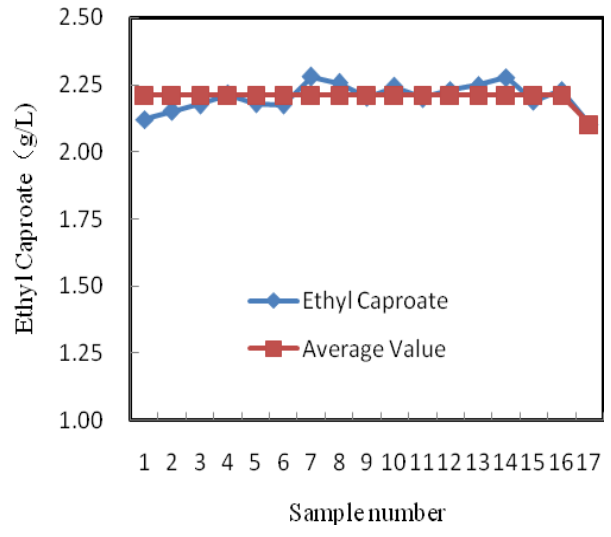

Figure 18. Ethyl caproate content trends. 


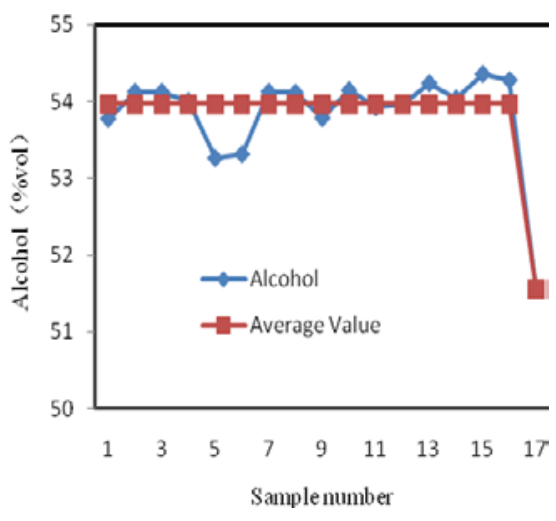

Figure 19. Alcohol content trends.

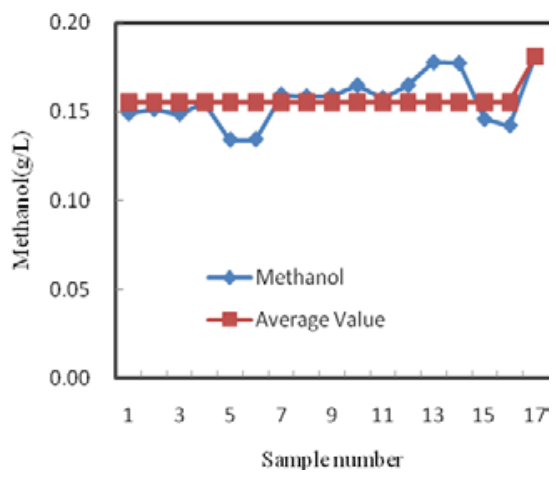

Figure 20. Methanol content trends.

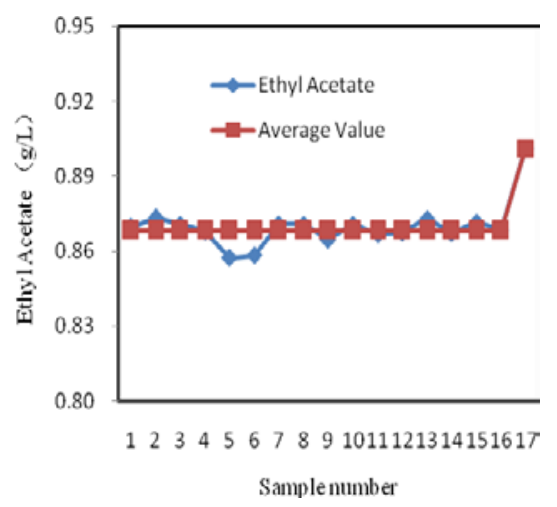

Figure 21. Ethyl acetate content trends.

the sample, and to the stability of judge, so that the established model has a wider and better application value; 2) The detection rate of fakes. Fakes detection rate to a certain extent depends on the wine samples and the true wine simulation is high or low, so the experiment to select fakes of simulation is higher.

\section{Acknowledgements}

The research was financially fitted by the "Doubles" focused on protection of geographic marks of white wine of origin inspection techniques (2012104019-2), Author acknowledge the support with gratitude.

\section{REFERENCES}

[1] L. Z. Chen, J. Zhao, Z. H. Ye, et al., "Determination of Adulteration in Honey Using Near-Infrared Spectroscopy,” Spectroscopy and Spectral Analysis, Vol. 11, No. 28, 2008, pp. 2565-2568.

[2] E. Corbella and D. J. Cozzolino, "Near Infrared Spectrosc,” Vol. 13, No. 2, 2005, p. 63.

[3] G. T. Xu, Z. L. Liu, Y. R. Yang, et al., "Determination of Esel Fuel Composition by Near Infrared Spectroscopy and its Application," Acta Pentrolei Sinica(Pentrolun processing Section), Vol. 8, 2002, pp. 65-71.

[4] M. Pardo, G. Sberveglieri, "Random Forests and Nearest Shrunken Centroids for the Classification of Sensor Array Data," Sensors and Actuators B, Vol. 131, No. 1, 2008, pp. 93-99. http://dx.doi.org/10.1016/j.snb.2007.12.015

[5] P. Jan, D. B. Bernard, E. C. Niko, "Random Forests as a Tool for Ecohydrological Distribution Modeling,” Ecological Modeling, Vol. 207, No. 2-4, 2007, pp. 304-318. http://dx.doi.org/10.1016/j.ecolmodel.2007.05.011

[6] X. Huang, W. Pan, S. Grindle et al., "A Comparative Study of Discriminating Human Heart Failure Etiology Using Gene Expression Profiles,” Bioinformatics, Vol. 6, No. 1, 2005, p. 205.

[7] I. Arana, C. Jarn and S. Arazuri, "Maturity, Variety and Origin Determination in White Grapes (Vitis vinifera L.) Using Near Infrared Reflectance Technology,” Journal of Near Infrared Spectroscopy, Vol. 13, No. 6, 2005, p. 349. http://dx.doi.org/10.1255/jnirs.566

[8] B. X. Li, Y. H. Wei, L. L. Xi, et al., "Qualitative Research on Radix Angelicae Sinensis from Different Origins in Different Harvest Time by Near Infrared Spectrum," Chinese Journal of Spectroscopy Laboratory, Vol. 4, No. 28, 2011, pp. 2128-2134.

[9] B. Chen, B. H. Jin, H. Liang, et al., "Identification of Chinese Tea by Near Infrared Reflectance Spectroscopy and Cluster Analysis," Chinese Journal of Spectroscopy Laboratory, Vol. 5, No. 29, 2012, pp. 2901-2905.

[10] D. H. Ma, X. C. Wang, L. P. Liu, et al., "Current Progress in Food Geographical Origin Traceability by Near Infrared Spectroscopy Technology,” Spectroscopy and Spectral Analysis, Vol. 4, No. 31, 2011, pp. 877-881.

[11] B. Y. Xia and Q. Ren, "Review on Some Methods for Date Process of Near Infrared Spectroscopy Analysis," Chinese Journal of Spectroscopy Laboratory, Vol. 3, No. 23, 2005, pp. 629-634.

[12] H. Qin, Z. J. Lin and J. W. Chen, "Partial Least-Squares Regression Theory, Analytical Steps and Procedures," Journal of Mathematical Medicine, Vol. 4, No. 20, 2007, pp. 450-451.

[13] Z. L. Ye, K. Yu and Y. Y. Cheng, "Near Infrared Chemical Fingerprinting Based on Wavelet Transform," Chemical Journal of Chinese Universities, Vol. 3, No. 28, 2007, pp. 441-444. 